\title{
Proteomics comparison of the sera from multiple sclerosis patients and neuromyelitis optica patients
}

\author{
S.F. Jiang ${ }^{1}$, Q.Y. Lu ${ }^{2}$, S. Hu², Y. Chen ${ }^{3}$, X.L. Liu ${ }^{4}$, Y. Yang ${ }^{1}$ and M.P. Ding ${ }^{1}$ \\ ${ }^{1}$ Department of Neurology, The Second Affiliated Hospital, School of Medicine, \\ Zhejiang University, Hangzhou, China \\ ${ }^{2}$ Air Force Centre of Aviation Medical Evaluation and Training in Hangzhou, \\ Hangzhou, China \\ ${ }^{3}$ Department of Geriatrics, The Second Affiliated Hospital, School of Medicine, \\ Zhejiang University, Hangzhou, China \\ ${ }^{4}$ Department of Neurology, Zhejiang Hospital, Hangzhou, China \\ Corresponding author: M.P. Ding \\ E-mail: meipingding@yeah.net
}

Genet. Mol. Res. 13 (4): 9292-9299 (2014)

Received May 21, 2013

Accepted December 18, 2013

Published February 14, 2014

DOI http://dx.doi.org/10.4238/2014.February.14.3

\begin{abstract}
This study aimed to identify the proteins that are differentially expressed in sera of multiple sclerosis (MS) patients, neuromyelitis optica (NMO) patients, and normal controls by using a two-dimensional gel electrophoresis (2-DE) assay. Serum samples were collected from the 3 groups, and total proteins were isolated and quantified by using the Bradford assay. The 2-DE and silver staining were carried out, and the Image Master 2D Platinum 5.0 software was used to analyze the images. Differentially expressed protein spots were removed from the gel and digested by enzymolysis and high-definition tandem mass spectrometry, and the MASCOT online software was applied to identify the proteins. Three differentially expressed proteins were identified: immunoglobulin (Ig) lambda chain, keratin 83, and haptoglobin (Hp2); the Ig lambda chain was only found in MS patients.
\end{abstract}


The expression of keratin 83 was significantly elevated in the MS group compared to the normal control or NMO groups. The expression of Hp2 in the NMO group was elevated by more than 2-fold compared to the normal control or MS groups. In summary, a variety of proteins were found to be differentially expressed in the serum between MS, NMO, and normal subjects using 2-DE: Ig lambda, Hp2, and keratin 83 were identified as potential diagnostic markers or treatment targets for MS or NMO.

Key words: Multiple sclerosis; Neuromyelitis optica; Proteomics

\section{INTRODUCTION}

Multiple sclerosis (MS) is an autoimmune inflammatory disease of the central nervous system (CNS). In MS, demyelination is characterized by inter-individual rather than intra-individual heterogeneity, with four distinct patterns of focal demyelination identified histologically in biopsy and autopsy samples. Neuromyelitis optica (NMO) is an idiopathic inflammatory demyelinating disease of the CNS that predominantly affects the optic nerves and the spinal cord (Matiello et al., 2007). NMO is a severe monophasic syndrome characterized by bilateral optic neuritis and myelitis that occur in rapid succession. Studies on serum NMO-immunoglobulin (Ig) G have indicated that its presence is highly specific to NMO (Lennon et al., 2004; Saiz et al., 2007). However, given that NMO-IgG is not detectable in all patients, the diagnosis of NMO is generally based on a combination of clinical, neuroimaging, laboratory, and pathological findings (Argyriou and Makris, 2008; Lana-Peixoto, 2008).

Although MS and NMO are both categorized as demyelinating diseases of the CNS, they differ in terms of their pathogenesis, pathology, clinical features, and treatment options (Bergamaschi et al., 2004; Lennon et al., 2005; Papeix et al., 2007; Lucchinetti, 2008; Hauser et al., 2008). Serum proteomics is an effective technique for monitoring serum protein expression. NMO and MS are characterized by abnormal serum findings; thus, proteomics might provide important information for deciphering the mechanisms underlying NMO and MS pathogenesis. In the present study, the differences in serum proteins of MS and NMO patients and normal controls were compared using two-dimensional gel electrophoresis (2-DE) to identify potential protein markers and their roles in pathogenesis. Understanding the expression and function of these disease-related proteins may allow us to identify these proteins as biomarkers to aid in diagnosing NMO and, potentially, in its treatment and prognosis.

\section{MATERIAL AND METHODS}

\section{Participants and preparation}

The selected MS patients satisfied the McDonald diagnostic criteria (2005 version) for MS and were all in the recurrent-remitting acute period. There were 2 male patients and 
4 female patients who ranged in age from 18 to 45 years, with a mean age of $28.7 \pm 9.6$ years. The selected NMO patients fulfilled the Wingerchuk diagnostic criteria for NMO. There were 3 male patients and 3 female patients who ranged in age from 21 to 39 years, with a mean age of $27.2 \pm 8.4$ years. The control group consisted of healthy subjects, 3 males and 3 females, who ranged in age from 19 to 35 years with a mean age of $25.2 \pm 6.2$ years. The subjects of the control group had no specific previous disorders and neurological examinations showed no pathological signs. The present study was conducted in accordance with the Declaration of Helsinki with approval from the Ethics Committee of the Second Affiliated Hospital, School of Medicine, Zhejiang University. Written informed consent was obtained from all participants.

Sera were collected with consent from the volunteers. After routine clinical tests, the remaining samples were placed in sterile centrifuge tubes and centrifuged at $2000 \mathrm{rpm}$ for $15 \mathrm{~min}$. After centrifugation, the supernatant fluid was collected and distributed in sterile EP tubes. Subsequently, the EP tubes were stored at $-80^{\circ} \mathrm{C}$ for later use.

\section{2-DE}

The first phase of the isoelectric focusing electrophoresis was conducted using the IPGphor IEF System. First, the protein was centrifuged for $2 \mathrm{~min}$ prior to sample loading. Then, a 100- $\mu \mathrm{g}$ sample, which was resolved with rehydration solution containing $8 \mathrm{M}$ urea, $0.02 \%$ CHAPS lysis buffer, $0.02 \mathrm{M}$ dithiothreitol (DTT), and $0.05 \%$ immobilized $\mathrm{pH}$ gradient buffering solution was loaded, followed by the addition of $800 \mu \mathrm{L}$ covering fluid. The focused strip was balanced twice in sodium dodecyl sulfate (SDS) balance solution (1.5 M Tris-HCl, pH 8.8, $50 \mathrm{mM} \mathrm{30 \%} \mathrm{glycerol,} 6 \mathrm{M}$ urea, 2\% SDS, and trace bromophenol blue), and was subsequently shaken twice on an oscillation bed for $15 \mathrm{~min}$. Then, 20 $\mathrm{mM}$ DTT was added to the first balance solution, whereas $100 \mathrm{mM}$ iodoacetamide was added to the second balance solution. After these procedures, we moved the strip to the PROTEAN II xi Cell for vertical SDS-polyacrylamide gel electrophoresis (PAGE) using a $13 \%$ polyacrylamide gel separator at a $40-\mathrm{mA}$ constant current for $40 \mathrm{~min}$ and a $60-\mathrm{mA}$ constant current for $5 \mathrm{~h}$. The process was only concluded once the front of the bromophenol blue reached the bottom of the glass. The electrophoresis was repeated three times for each group of samples.

\section{Silver staining}

Silver staining was conducted as shown in Table 1, following instructions of the SilverQuest Silver Staining Kit manual (Invitrogen). After electrophoresis, the gel was placed in a clean, appropriately sized staining tray. The gel was rinsed briefly with ultrapure water and subsequently fixed in $100 \mathrm{~mL}$ fixative solution $(40 \%$ ethanol and $10 \%$ acetic acid in ultrapure water) for $30 \mathrm{~min}$ with gentle rotation. Afterward, the fixative solution was decanted and the gel was washed in $30 \%$ ethanol for $10 \mathrm{~min}$, followed by sensitization in sensitizing solution for $30 \mathrm{~min}$. Subsequently, the gel was washed and incubated in $100 \mathrm{~mL}$ staining solution for $20 \mathrm{~min}$ to react with silver. Finally, the gel was washed again and placed in developing solution until spots appeared on the gels. Stopper solution was then added for 10 min to terminate the reaction (Table 1). 


\begin{tabular}{ll} 
Table 1. Silver staining. \\
\hline Steps & Time \\
\hline 1. Stationary liquid & $30 \mathrm{~min}$ \\
2. Sensitizer & $30 \mathrm{~min}$ \\
3. Washing & $3 \times 5 \mathrm{~min}$ \\
4. Silver reaction & $20 \mathrm{~min}$ \\
5. Washing & $2 \times 1 \mathrm{~min}$ \\
6. Staining & $2-5 \mathrm{~min}$ \\
7. Terminator & $10 \mathrm{~min}$ \\
\hline
\end{tabular}

\section{Scanning and image analysis}

First, the gel was scanned using an image scanner, saved in TIFF format, and then analyzed using the ImageMaster 2D Platinum 5.0 software. Then, the test parameters, particularly the smooth optimization parameters, were set to detect all real protein spots and to split overlapping protein spots. We were able to filter the interference noise and finish the automatic detection (spot detection), as well as quantify and remove the background by adjusting the saliency and minimum area. Manually spot marking the gel allowed us to increase or decrease the number of spots and to copy and split spots to obtain a protein spot detection map. We then determined the molecular length and molecular weight of the protein spots by marking the marker protein and $\mathrm{pH}$. Finally, the reference gel was selected for comparison with other gels to obtain a gel analysis report. The volume percentage of a specific spot to the total gel was calculated, and the differences among protein spots were compared through statistical analysis using the between group Student $t$-test. We differentiated the protein spots based on whether their analytical differences were significant and whether their volume percentages were twice as high.

\section{Slicing of the gel sample}

The tips of $200 \mu \mathrm{L}$ yellow pipettors were cut into equal sizes as much as possible and immersed in washing lotion for 1 day. Afterward, the pipettors were sequentially immersed in distilled water and redistilled water and ultrasonicated. The gel samples were thoroughly washed with redistilled water and baked until dry. The protein of interest was either pulled from the gel with cut gun tips or cut with a $1.5-\mathrm{mm}$ gel pen and placed in EP tubes and on 96well plates. The number of spots and their corresponding positions were recorded.

\section{Enzymatic digestion}

The gel was washed twice with $50 \mu \mathrm{L} \mathrm{ddH}_{2} \mathrm{O}$ for $10 \mathrm{~min}$. Then, $50 \mu \mathrm{L}$ acetonitrile was added to dehydrate the gel until it turned completely white. The gel was subjected to a vacuum for $5 \mathrm{~min}$. Then, $20 \mu \mathrm{L} 10 \mathrm{mM}$ DTT $\left(10 \mu \mathrm{L} 1 \mathrm{M}\right.$ DTT in $\left.990 \mu \mathrm{L} 25 \mathrm{mM} \mathrm{NH}_{4} \mathrm{HCO}_{3}\right)$ was added and heated in a $56^{\circ} \mathrm{C}$ water bath for $1 \mathrm{~h}$. After cooling to room temperature, the gel was dried and $20 \mu \mathrm{L} 55 \mathrm{mM}$ iodoacetamide (IAA) $\left(55 \mu \mathrm{L} 1 \mathrm{M}\right.$ IAA in $\left.945 \mu \mathrm{L} 25 \mathrm{mM} \mathrm{NH}_{4} \mathrm{HCO}_{3}\right)$ was quickly added and kept in a darkroom for $45 \mathrm{~min}$. The gel particles were subsequently washed with 25 $\mathrm{mM} \mathrm{NH} \mathrm{HCO}_{3}, 50 \%$ acetonitrile solution, and acetonitrile, and they were subsequently dehydrated using acetonitrile until they turned completely white. The gel particles were then vacuumdried for $5 \mathrm{~min}$. Then, stock $0.1 \mu \mathrm{M}$ trypsin solution was diluted 10 -fold using $25 \mathrm{mM} \mathrm{NH}_{4} \mathrm{HCO}_{3}$. Furthermore, $2 \mu \mathrm{L}$ dilution was added into each EP tube. Then, the tubes were centrifuged for a 
short time to ensure that the gel particles were fully mixed with the enzyme. The EP tubes were either kept at $4^{\circ} \mathrm{C}$ or placed on ice for 30 min until the solution was completely absorbed by the gel particles. Then, $25 \mathrm{mM} \mathrm{NH}_{4} \mathrm{HCO}_{3}$ was added into each tube to a total volume of 10-15 $\mu \mathrm{L}$. The tubes were then incubated overnight at $37^{\circ} \mathrm{C}$ to allow digestion. The following day, $2 \%$ triflouroacetic acid (TFA) was added to the tubes to stop the reaction, and the final TFA concentration was maintained at $0.1 \%$. Finally, the mixture was shaken and centrifuged.

\section{High-definition tandem mass spectrometry (HD-MS/MS) analysis}

After digestion, the enzyme hydrolysate samples were loaded to a $1-\mu \mathrm{L} 300$-well stainless steel plate. Moreover, the same sample loading procedure was repeated two more times after the first sample spots dried. Consequently, $1 \mu \mathrm{L}$ substance (cyano-4-hydroxycinnamic acid) was added. Then, $1.1 \mu \mathrm{L}$ calibration mixture was added in the middle of the samples for calibration while loading. The peak of trypsin self-hydrolysate was used for internal calibration. The model was left to dry at room temperature and was subsequently sent to the Beijing Military Medical Sciences Mass Spectrometry Laboratory for HD-MS/MS analysis using a Synapt HD-MS produced by Waters Micromass Corporation.

\section{Database search}

The search was accomplished using the MASCOT software, which can be found at the following online protein database: http://www.mastrix-science.co.uk.

\section{Preliminary analysis of protein spots}

The featured proteins were searched and identified based on their isoelectric point and relative molecular mass from the gel analysis using an online protein search site (http://www. expasy.ch/swiss-2dpage/viewer). The proteins were then compared with the 2-D-PAGE serum reference map of the SWISS-2DPAGE database. The preliminary analysis of these proteins was performed using relevant literature.

\section{RESULTS}

The serum 2-D gel electrophoresis map revealed 414 points in the MS group, 472 points in the NMO group, and 476 points in the normal control group (Figure 1).

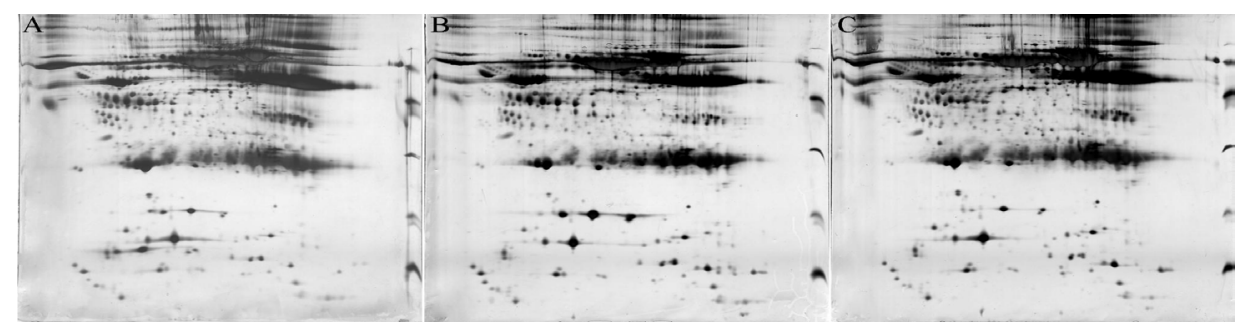

Figure 1. Serum 2-D gel electrophoresis map (after analysis). A. MS group. B. NMO group. C. normal control group. 
Comparison of the serum 2-DE patterns of the MS group and the control group revealed 345 matched protein spots. Among the 345 matched spots, the MS group had 159 upregulated proteins compared with the normal group, 42 of which were upregulated by at least 2 -fold. Furthermore, there were 186 downregulated protein spots. There were 69 protein spots that did not match the MS group and 131 spots that did not match the normal control group.

Comparison of the serum 2-DE patterns of the NMO group and the control group revealed 374 matched protein spots. Among the 374 matched spots, the NMO group had 175 upregulated proteins compared with the normal group, 57 of which were upregulated by at least 2 -fold. Furthermore, there were 199 downregulated protein spots. There were 98 protein spots that did not match the NMO group and 102 spots that did not match the normal control group.

Up to 52 serum protein spots were expressed in the MS and control groups that were not expressed in the NMO group. Furthermore, 81 serum protein spots were expressed in the control and NMO groups that were not expressed in the MS group.

The HD-MS/MS analysis and MASCOT web search identified three differentially expressed proteins: Ig lambda, haptoglobin (Hp2), and keratin 83. Ig lambda was only found in the serum 2-DE map of MS patients. The keratin 83 expression level in the MS group was at least 2-fold higher than those in the normal control and NMO groups. The Hp2 expression levels in the NMO group were at least 2-fold higher than those in the normal control and MS groups (Table 2).

\begin{tabular}{|c|c|c|c|c|}
\hline Spot & Protein name & Accession No. & Experimental mass/pI & MASCOT score \\
\hline MS1063 & Ig lamda chain & gi|106638 & $24733 / 6.71$ & 391 \\
\hline MS1163 & Keratin 83 & gi $\mid 169790841$ & $54161 / 5.54$ & 350 \\
\hline NMO1192 & Haptoglobin (Hp2) & gi|223976 & $41717 / 6.23$ & 918 \\
\hline
\end{tabular}

\section{DISCUSSION}

Research on the serum proteomics of MS is limited. Comparative studies on the latent membrane protein in the sera of MS patients and normal subjects were carried out using flight mass spectrometry and tandem mass spectrometry through matrix-assisted laser desorption ionization. The results showed that the serum concentration of complement fragments was related to MS morbidity, which demonstrated that this fragment is a potential diagnostic marker for MS in the serum (Sawai et al., 2010). In an analysis of plasma samples of pediatric MS patients and normal subjects using 2-DE combined with mass spectrometry, Rithidech et al. (2009) found that the serum concentrations of 12 types of albumen were significantly higher among MS patients.

In the present study, serum 2-DE was performed in samples from MS patients, NMO patients, and normal controls. The image analysis software and analysis of serum proteins identified three proteins: the Ig lambda chain, keratin 83, and Hp2. The Ig lambda chain was expressed in the serum of MS patients, but not in the normal group, which implied that the Ig lambda chain is expressed at a higher level in MS serum. Keratin 83 was expressed in the MS group and in normal controls, although the expression level in the MS group was up to 13 times higher than that in the normal controls. The serum Hp2 level of NMO patients was three times higher than that of the normal controls. 
Immunoglobulin consists of two identical heavy chains and two identical light chains, which can be classified as $\kappa$ and $\lambda$ (Butler et al., 2009). The classical viewpoint proposes that MS is a myelin-sensitive self-activated $\mathrm{CD} 4^{+} \mathrm{T}$ cell-mediated autoimmune disease because activated myelin $\mathrm{CD}^{+} \mathrm{T}$ cells were shown to induce experimental autoimmune encephalomyelitis in animal models. Although many studies have focused on myelin-specific $\mathrm{CD}^{+} \mathrm{T}$ cells, their function is still unclear. Evidence of B cell involvement in the progression of MS has increased in recent years. The continuous IgG synthesis in the cerebrospinal fluid and the generation of oligoclonal bands is an important immunological index that indicates the participation of humoral immunity in the pathophysiology of MS. Antibodies may participate in the activation of the demyelination of MS patients. A previous study also found that the fundamental pathological changes in MS patients exhibited damage to the active myelin area IgG and the deposition of the activated complement (Noseworthy, 1999). Moreover, Genain et al. (1999) demonstrated that the serum and myelin basic protein autoantibodies in the cerebrospinal fluid of MS patients have different levels of escalation. Myelin basic protein antibodies and myelin oligodendrocyte glycoprotein have been isolated from MS nidi and immunoglobulin, and complement depositions have been demonstrated in the myelin degradation area of MS nidi (Genain et al., 1999). These findings demonstrate the involvement of humoral immunity in the occurrence and development of MS. The high serum expression of the $\operatorname{Ig} \lambda$ chain also supports this conclusion. However, further studies are required to determine whether the high Ig $\lambda$ chain expression is related to damage to the blood-brain barrier.

As an acute phase protein, haptoglobin participates in host resistance to infection, the repair of damaged tissue, and processes of the internal environment. Furthermore, haptoglobin has antioxidative activity, inhibits prostaglandin synthesis, bacterial growth, and nitrous oxide synthesis, promotes angiogenesis, and adjusts the immune function.

Haptoglobin inhibits the plant hemagglutinin-mediated lymphocyte transformation, which is positively correlated with serum haptoglobin density. Haptoglobin also inhibits the lymphocyte transformation caused by different types of agglutinin (Langlois and Delanghe, 1996). Haptoglobin also regulates the Thl/Th2 balance (O'Bryan et al., 1997). In vitro, haptoglobin inhibits the release of Th2 cell factors (IL-4, IL-5, IL-10, and IL-13), mildly inhibits the synthesis of Th1 cell factors, IFN- $\gamma$ and IL-2, and functions in immune balance (Butler et al., 2009). Wagner et al. (1996) found that haptoglobin regulates the activity of neutrophilic granulocytes and mononuclear cells to regulate the immune response; thus, haptoglobin may function as an immune receptor and as natural antagonists of ligand activation. A proteomic study among patients with fronto-temporal dementia demonstrated significantly decreased haptoglobin expression in the cerebrospinal fluid (Hansson et al., 2004). Haptoglobin levels are significantly higher in the brain tissue and cerebrospinal fluid of Alzheimer's disease patients (Davidsson et al., 2002). The change in haptoglobin expression is possibly a result of local inflammation after the acute phase of the protein change and immune function, which may indicate its function in the NMO immune response.

Further studies are needed to determine the role of keratin 83 in the pathogenesis of MS pathogenesis.

\section{REFERENCES}

Argyriou AA and Makris N (2008). Neuromyelitis optica: a distinct demyelinating disease of the central nervous system. Acta Neurol. Scand. 118: 209-217. 
Bergamaschi R, Tonietti S, Franciotta D, Candeloro E, et al. (2004). Oligoclonal bands in Devic's neuromyelitis optica and multiple sclerosis: differences in repeated cerebrospinal fluid examinations. Mult. Scler. 10: 2-4.

Butler JE, Zhao Y, Sinkora M, Wertz N, et al. (2009). Immunoglobulins, antibody repertoire and B cell development. Dev. Comp. Immunol. 33: 321-333.

Davidsson P, Sjogren M, Andreasen N, Lindbjer M, et al. (2002). Studies of the pathophysiological mechanisms in frontotemporal dementia by proteome analysis of CSF proteins. Brain Res. Mol. Brain Res. 109: 128-133.

Genain CP, Cannella B, Hauser SL and Raine CS (1999). Identification of autoantibodies associated with myelin damage in multiple sclerosis. Nat. Med. 5: 170-175.

Hansson SF, Puchades M, Blennow K, Sjogren M, et al. (2004). Validation of a prefractionation method followed by two-dimensional electrophoresis - Applied to cerebrospinal fluid proteins from frontotemporal dementia patients. Proteome Sci. 2: 7.

Hauser SL, Waubant E, Arnold DL, Vollmer T, et al. (2008). B-cell depletion with rituximab in relapsing-remitting multiple sclerosis. N. Engl. J. Med. 358: 676-688.

Lana-Peixoto MA (2008). Devic's neuromyelitis optica: a critical review. Arq. Neuropsiquiatr. 66: 120-138.

Langlois MR and Delanghe JR (1996). Biological and clinical significance of haptoglobin polymorphism in humans. Clin. Chem. 42: 1589-1600.

Lennon VA, Wingerchuk DM, Kryzer TJ, Pittock SJ, et al. (2004). A serum autoantibody marker of neuromyelitis optica: distinction from multiple sclerosis. Lancet 364: 2106-2112.

Lennon VA, Kryzer TJ, Pittock SJ, Verkman AS, et al. (2005). IgG marker of optic-spinal multiple sclerosis binds to the aquaporin-4 water channel. J. Exp. Med. 202: 473-477.

Lucchinetti C (2008). Pathological heterogeneity of idiopathic central nervous system inflammatory demyelinating disorders. Curr. Top. Microbiol. Immunol. 318: 19-43.

Matiello M, Jacob A, Wingerchuk DM and Weinshenker BG (2007). Neuromyelitis optica. Curr. Opin. Neurol. 20: 255260.

Noseworthy JH (1999). Progress in determining the causes and treatment of multiple sclerosis. Nature 399: A40-A47.

O'Bryan MK, Grima J, Mruk D and Cheng CY (1997). Haptoglobin is a Sertoli cell product in the rat seminiferous epithelium: its purification and regulation. J. Androl. 18: 637-645.

Papeix C, Vidal JS, de Seze J, Pierrot-Deseilligny C, et al. (2007). Immunosuppressive therapy is more effective than interferon in neuromyelitis optica. Mult. Scler. 13: 256-259.

Rithidech KN, Honikel L, Milazzo M, Madigan D, et al. (2009). Protein expression profiles in pediatric multiple sclerosis: potential biomarkers. Mult. Scler. 15: 455-464.

Saiz A, Zuliani L, Blanco Y, Tavolato B, et al. (2007). Revised diagnostic criteria for neuromyelitis optica (NMO). Application in a series of suspected patients. J. Neurol. 254: 1233-1237.

Sawai S, Umemura H, Mori M, Satoh M, et al. (2010). Serum levels of complement C4 fragments correlate with disease activity in multiple sclerosis: proteomic analysis. J. Neuroimmunol. 218: 112-115.

Wagner L, Gessl A, Parzer SB, Base W, et al. (1996). Haptoglobin phenotyping by newly developed monoclonal antibodies. Demonstration of haptoglobin uptake into peripheral blood neutrophils and monocytes. J. Immunol. 156: 1989-1996. 